\title{
Effects of absolute mask energy and mask energy range on metacontrast suppression
}

\author{
IRA H. BERNSTEIN and SEBASTIANO A. FISICARO \\ University of Texas at Arlington, Arlington, Texas 76019
}

\begin{abstract}
In an experiment similar to one by Bernstein, Fisicaro, and Fox (1976), subjects were asked to judge the brightness of test stimuli in a metacontrast display. The energies of the test and of the mask varied orthogonally. Various experimental conditions differed with respect to the range of mask energies. Individually, the mask energies were generally weaker than the test energies. The results replicated the main findings of Bernstein et al. (1976) in that judgments of the test were negatively correlated with mask energy at short stimulus onset asynchrony (SOA) but positively correlated at long SOA. These effects were more pronounced when the masks were similar in energy range and level to the tests than when they were wider in energy range and weaker. In general, there was no evidence for the effects of trial-to-trial variation described by Hake, Faust, McIntyre, and Murray (1967).
\end{abstract}

We have previously examined the role of judgmental factors in metacontrast (Bernstein, Fisicaro, \& Fox, 1976; Bernstein, Proctor, Belcher, \& Schurman, 1974; Bernstein, Proctor, Proctor, \& Schurman, 1973; Bernstein, Smith, \& Adey, in press). The premise of these studies was that the mask introduces new cues whose effects vary with stimulus onset asynchrony (SOA). At very short SOAs, the mask may aid the judgmental process by serving as a frame of reference to stabilize judgments. At very long SOAs, the mask plays little role in judgments about the test because the subject treats the two events separately. However, at those SOAs producing suppression, the mask misinforms the subject, who perceives the fading trace of the test as a less intense event presented at a shorter SOA. This general point of view is a broader conceptualization of masking than that stated by Breitmeyer and Ganz (1976), Weisstein (1972), and others who attribute suppression to the destruction of information through lateral inhibition. It also emphasizes how subjects pay attention to different features of their stimulus environment as SOA changes.

Bernstein et al. (1976) presented data in support of the latter assertion. They had subjects identify various tests whose energies and those of the mask varied orthogonally. Three energy levels were used for both tests and masks (i.e., bright, medium, and dim tests

This study was supported in part through funds provided by the Department of Ophthalmology, University of Texas Health Science Center at Dallas, Dallas, Texas 75225, where the senior author is also affiliated, the Organized Research Fund of the University of Texas at Arlington, and the Genco Abbandando Foundation. Requests for reprints should be addressed to Ira $\mathrm{H}$. Bernstein, Department of Psychology. University of Texas at Arlington, Arlington, Texas 76019. and masks), which were separated by $.2 \log$ unit in energy (luminance $x$ duration). Pilot data indicated that the test alternatives could be discriminated with an accuracy of approximately $70 \%$ in the absence of a mask. On experimental trials, the SOA was held constant within blocks and was varied from 0 to 180 msec between blocks. "No-mask" control trials were also included.

The design allowed both judgmental accuracy and, more importantly, the structure underlying these judgments to be explored. The linear discriminant function (LDF), whose role in the study of perception was described by Hake (Hake, Faust, McIntyre, \& Murray, 1967; Hake \& Rodwan, 1966; Hake, Rodwan, \& Weintraub, 1966; Rodwan \& Hake, 1964), was employed to serve these ends. Mathematically, the LDF is that linear combination of predictors (test energy and mask energy) which maximally discriminates group means (response categories).

Both the accuracy and the coherence (consistency) of judgments were U-shaped functions of SOA, paralleling what is normally obtained by traditional methods which do not allow inferences about the subject's decision structure. Consistent with the view that the mask serves as a frame of reference, accuracy at 0-msec SOA was superior to accuracy in the "no-mask" control condition.

The slope of the LDF was used as a partial definition of the subject's criterion content. Kahneman (1968) used the latter term to describe the code used by subjects to map their experiences onto responses. The slopes of the LDF were negative at 0 -msec SOA. Hence, the code consisted of the difference between test energy and mask energy. This illustrates simultaneous contrast: the test was perceived as dimmer when either its energy was decreased or when the 
energy of the surround (mask) was increased. Hence, it can be interpreted as a consequence of lateral inhibition. However, lateral-inhibitory theories indicate that such effects should increase with SOA, at least to the point of maximum suppression. Bernstein et al.'s (1976) findings were that the correlation (i.e., slope of the LDF) decreased as SOA increased. In fact, when accuracy was at its minimum, judgments of the test were uncorrelated with mask energy. Furthermore, as SOA increased past this point, the correlation between judgments of the test and mask energy became positive, i.e., subjects responded to the sum of test and mask energies. This effect decreased as SOA increased still further, and, as one might expect, mask energy played essentially no role in judgments made at the longest SOA.

The distribution of responses made to the various stimulus combinations were compared to the distributions predicted by two judgmental models presented in Hake et al. (1967): the nondiscrimination observer model and the discriminating observer model. The former assumes that the LDF contains all the information needed to make decisions. This implies that subjects treat test brightness and mask brightness as a single, or integral (Garner, 1974), dimension and that brightness judgments are determined by operations performed at a single, processing stage. However, the data were better described by the discriminating observer model. This assumes that subjects treat test brightness and mask brightness as separable dimensions. Thus, their judgment space is two-dimensional, and two processing stages are involved. One processing stage is used to encode the two energies into brightnesses, and the other is used to combine the brightnesses to form a judgmental axis.

The experiment to be reported is a replication and extension of the Bernstein et al. (1976) study. The same general procedure, including the set of test energies, was employed. In one condition, serving as the replication, the same set of mask energies was used, i.e., $.0,-.2$, and $-.4 \log$ units relative to the bright test. In the two remaining conditions, the sets of mask energies were $.0,-.4$, and $-.8 \log$ units and $.0,-.8$, and $-1.6 \log$ units, again referenced to the bright test.

One purpose of the study was to examine how the range of trial-to-trial variation of an irrelevant dimension affects performance. This was also the purpose of the Hake et al. (1967) study, which led our research in its current direction. They had subjects judge the size of a test square which appeared within an irrelevant, outer square in various experimental conditions or by itself in a control condition. The size of the outer square either remained constant or varied in size over different ranges. The basic finding was that performance was best when the outer square remained constant and was poorest when the outer square varied most. Hence, subjects did not ignore the outer square. The source of the decrement was not the same for all subjects: two behaved as nondiscriminating observers and responded progressively more to the outer square as its variation increased. One behaved as a discriminating observer and did not exhibit this change in criterion content.

The second purpose of this study was to examine possible suppressive effects when the mask is of lower energy than the test. The effects of mask energy, holding test energy constant, have been studied numerous times [see Lefton (1973) and Weisstein $(1968,1972)$ for pertinent reviews]. Normally, though, the mask is of equal or higher energy than the test. Under these conditions, the effects of increasing mask energy are: (a) to increase the amount of suppression, and (b) to decrease the SOA at which suppression is at a maximum. Typically, the masking function is monotonic when the mask is at least $1.0 \log$ unit more intense than the test.

In one rather extensive experiment, Alpern (1953) reported data obtained with both weak and strong masks. He found relatively minor and nonsystematic shifts in the point of maximum suppression. Though he did not present inferential data, some masking was apparently present when the mask was $1.0 \mathrm{log}$ unit less intense than the test.

\section{METHOD}

\section{Subjects}

Four graduate students enrolled at the University of Texas at Arlington were paid $\$ 2.30 / \mathrm{h}$ to serve as subjects. All had either normal or correct-to-normal eyesight.

\section{Apparatus and Stimuli}

Stimuli were presented by transillumination on a four-channel Iconix tachistoscope (Model 6192) using Sylvania bulbs (F8TS/D). Viewing was monocular through a 2-mm artificial pupil. A Spectra spotmeter (Model UBD-1) was used to verify luminance levels, and a Tektronix oscilloscope (Model 564) was used to verify timing accuracy. A telegraph key was used in order for subjects to selfinitiate trials after both experimenters indicated that the necessary adjustments had been made. The two experimenters and the subject sat in the same room.

A small circle that remained on continually was used for fixation. It was contained in Exposure Field IV but illuminated by a separate tungsten source which did not affect the other contents of the field. The fixation circle was sufficiently dim so as to be barely visible to a light-adapted subject. It became clear with dark adaptation and, for this reason, served as a partial control over adaptation effects.

Exposure Field I was used for the three alternative test stimuli (TS)-bright TS, medium TS, and dim TS. Each square appeared $.51 \mathrm{deg}$ of visual angle above the fixation circle. Exposure Fields II, III, and IV housed the three alternative masking stimuli (MS), each of which consisted of a pair of squares whose sides were adjacent to the TS. The length of each side of TS and MS was $1.0 \mathrm{deg}$ of visual angle. The location of each MS with respect to the three exposure fields was changed during the experi- 
ment. This was done to prevent coloration and other cues specific to each field from providing information.

The TS and MS durations were both $50 \mathrm{msec}$. The three TS were changed manually by one of the two experimenters by dropping Kodak neutral density filters into Exposure Field 1. The other experimenter both selected the MS appropriate for that trial by means of a toggle switch, which enabled one of the three exposure fields (II, III, or IV) to fire, and recorded the subject's responses on an answer sheet.

Luminances for bright TS, medium TS, and dim TS were 64,40 , and $25 \mathrm{~cd} / \mathrm{m}^{2}$, respectively (i.e., $.0,-.2$, and $-.4 \mathrm{log}$ units referenced to the bright TS). Three luminance range conditions were utilized in which the bright MS, medium MS, and $\operatorname{dim}$ MS luminances were: (a) $.0,-.2$, and $-.4 \log$ units (as in Bernstein et al., 1976) in the narrow-range condition, (b) .0, -.4 , and $-.8 \log$ units in the medium-range condition, and (c) $.0,-.8$, and $-1.6 \mathrm{log}$ units in the wide-range condition. These figures are referenced to the most intense TS and MS $\left(64 \mathrm{~cd} / \mathrm{m}^{2}\right)$.

As may be seen, the actual range of luminances (energies) covaries with both the mean mask energy and the weakest mask energy within the set. Thus, the narrow-range condition has a mean mask energy of -.2 and a weakest mask of -.4 ; the medium-range condition has a mean mask energy of -.4 and a weakest mask of -.8 ; and the wide-range condition has a mean mask energy of -.8 and a weakest mask of -1.6 . In Hake et al.'s (1967) experiment, their analogous manipulation of the dimensions of the outer square necessarily resulted in a covariation of range and the size of the smallest (as well as the largest) outer square in a set, but did not result in a covariation of range and the mean size within the set. The confounding in the present study arose because of the ceiling on test and mask energy. The most intense stimuli were jointly limited by maximum possible luminance available in our tachistoscope and the need to remain within the critical duration for temporal integration. Although we could have used a less intense set of tests and, thus, used both weaker and stronger masks, paralleling Hake et al. (1967), this did not seem wise. This is because it is well known that the magnitude of nonmonotonicity of a masking function is directly related to test energy for the equal-energy case. Hence, reducing the set of test energies would have made the basic effect at issue, U-shaped masking, more difficult to obtain. The analysis to be reported below will serve to separate luminance range and mean luminance. Thus, the term "luminance range" will still be used in a descriptive sense.

\section{Procedure}

The subjects first participated in several practice sessions during which they judged the brightness of the three TS energies alone (i.e., no mask) until their accuracy had apparently reached asymptote. The accuracies in terms of percent correct responses at the end of practice ranged from $56 \%$ to $69 \%$ and were within binomial error of one another for two consecutive sessions. At the beginning of each experimental session, the subjects were dark-adapted for $10 \mathrm{~min}$, after which they received 30 warm-up trials on TS alone with feedback. These data were not used further.

During the experiment, 270 trials were run at each of five SOAs $(0,30,60,120$, and $240 \mathrm{msec})$ under each of the three luminance ranges. Thus, the five SOAs and the three luminance ranges combined to form the experimental conditions. The 270 experimental trials per SOA in each luminance range condition were divided into three 90 -trial blocks. Within each experimental block, each of the nine stimulus alternatives ( 3 TS energies $\times 3$ MS energies) appeared 10 times. Likewise, the 90 control trials were divided into three 30-trial blocks. Within each control block, each of the three TS energies appeared 10 times.

One 90-trial block was run at each SOA to form a cycle of five blocks. Within the cycle, the five blocks were run in random order. Luminance range was held constant within the cycle. This process was repeated to form two additional cycles. Different randomizations were used within each cycle and across subjects.
Thus, there were three cycles per luminance range. All three cycles within a particular luminance range condition were completed before the subject was moved to another luminance range. However, each subject received the three luminance range conditions in a different order. During the last session of each of the three luminance range conditions, the subject received one of the three 30-trial control blocks (i.e., no mask). ${ }^{1}$ One cycle was run per session so that each subject participated in nine experimental sessions ( 3 cycles $\times 3$ luminance ranges).

The experimenters began each trial with appropriate dial settings and switch adjustments, including dummy dial settings and filter drops to avoid giving information to the subject. One experimenter then signaled the subject to initiate the trial, whereupon the subject responded "bright", "medium", or "dim" with respect to the apparent intensity of the TS. A rest break was given after each block.

\section{RESULTS}

Two sets of analyses were performed on the data. In the first, masking functions were compared for specific masks. In the second, LDF analyses were performed within each context. Except when noted, the group data describe individual subjects.

\section{Individual Masking Functions}

Two measures were obtained from the subject's judgments of test alternatives, conditional upon the particular mask that appeared on that trial. One was the percentage of correct responses, and the other was the mean response level ("bright" $=1$, "medium" = 2, "dim" = 3). These will be termed the accuracy and the bias measures, respectively." This accuracy measure, applicable to a single mask energy, should not be confused with the LDF accuracy measure which is applicable to a set of mask energies.

For each measure, nine masking functions may be obtained (3 luminance ranges $\times 3$ MS energies). A preliminary analysis ( 9 masks by 5 SOAs by 4 subjects) indicated that the main effects and the interactions were significant (all ps $<.01$ ) for both measures, except for the main effect of mask on the bias measure (n.s.)

Though this indicates that the nine masking functions differ, it is of limited utility because of the ways in which the masks might differ. Part of the effect could arise from differences between masking functions derived from masks of the same energy appearing in different contexts, e.g., .0 mask in the narrowrange condition vs. the .0 mask in the medium-range condition. Other effects could be due to differences between masks of different energy appearing in the same context, e.g., the .0 mask vs. the -.2 mask in the narrow-range condition. Finally, still other effects could be due to differences between masks of different energy appearing in different contexts, e.g., the .0 mask in the narrow-range condition vs. the -.8 mask in the medium-range condition. Con- 
sequently, additional analysis were performed comparing subsets of specific masks.

In the first set of analyses, purely contextual effects were examined by comparing the same MS energies in different contexts. Three such analyses were conducted: (a) the .0 mask in the narrow-, medium-, and wide-range conditions, (b) the -.4 mask in the narrow- and medium-range conditions, and (c) the -.8 mask in the medium- and wide-range conditions. In each of the three analyses of the accuracy measure, the masking functions did not differ from one another. Differences were noted for the bias measure in all three cases ( $p s<.01$ ). The functions were flatter when the particular mask was the lowest energy in the set as compared to when it was the middle or highest energy in the set. One implication of this finding is that the accuracy measure is freer from contextual effects than the bias measure.

The accuracy measures and the bias measures were then averaged over the different contexts (luminance ranges) in which they appeared in order to provide a single masking function for each of the five differ-

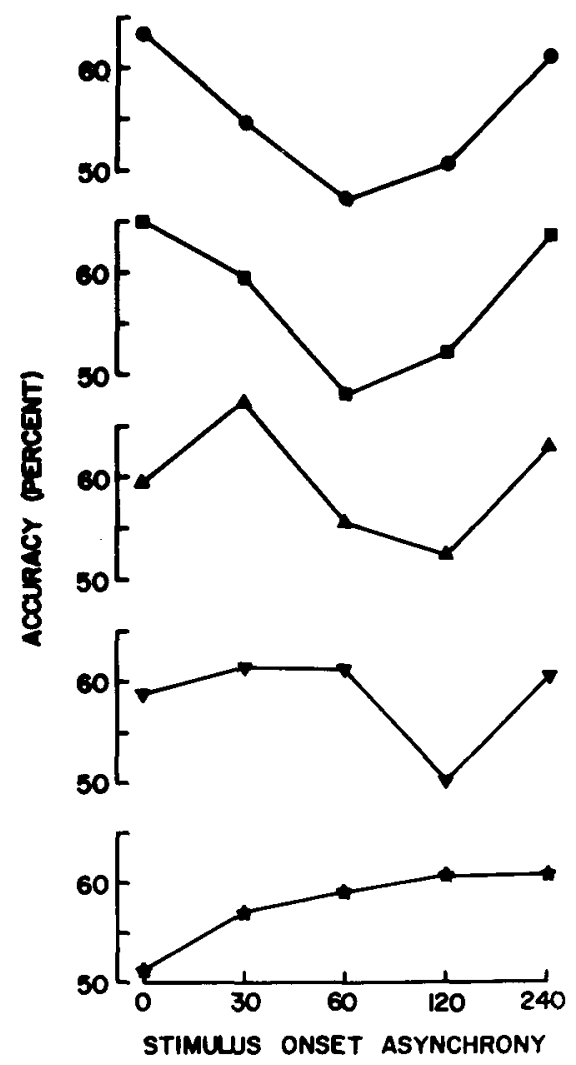

Figure 1. Accuracy (expressed in terms of the mean percentage of correct responses) as a function of absolute mask energy and stimulus onset asynchrony. (Note-From top to bottom, the masking functions correspond to absolute mask energies of .0 , $-.2,-.4,-.8$, and $-1.6 \log$ units referenced to the most intense test energy.)

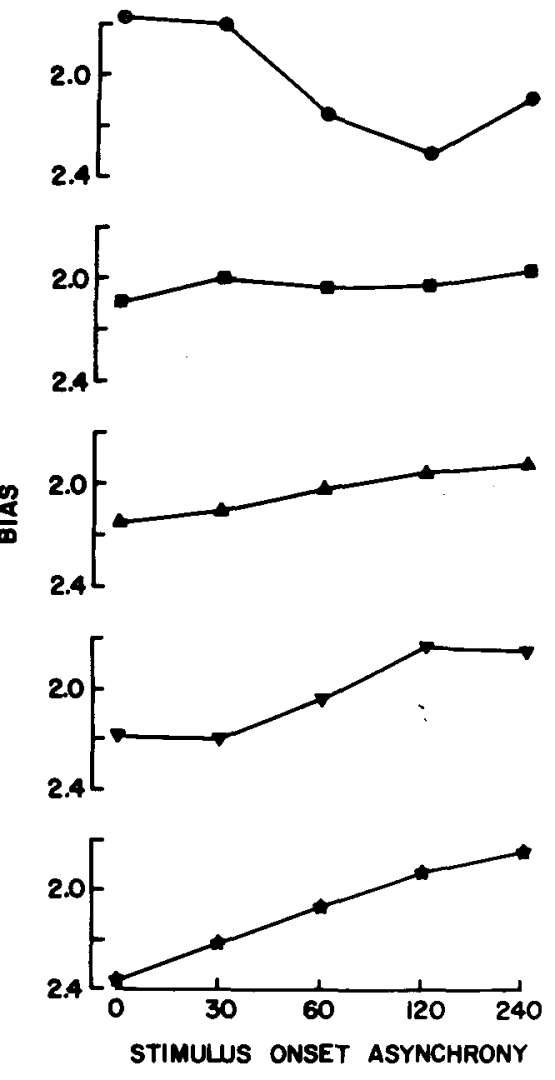

Figure 2. Bias (expressed in terms of the mean response level, with "bright" $=1$, "medium" = 2 , and "dim" $=3$ ) as a function of absolute mask energy and stimulus onset asynchrony.

ent MS energies $(.0,-.2,-.4,-.8$, and -1.6$)$. An analysis of variance of these five masking functions indicated that they were different. The F-ratios for the accuracy measure were $3.86(\mathrm{df}=4,12)$ and 2.77 ( $\mathrm{df}=16,48$ ) for the main effect of SOA and the interaction between SOA and energy, ps $<.05$ and .01 , respectively. The corresponding main effect and interaction $F$ ratios for the bias measure were 10.16 and 6.13 , ps $<.01$. The main effect of energy was nonsignificant as measured by both accuracy and bias, $\operatorname{Fs}(4,12)=2.27$ and $<1.0$, respectively.

The accuracy and bias masking functions are portrayed in Figures 1 and 2, respectively, and the results of trend tests are presented in Table 1. The accuracy functions for the .0 and -.2 MS energy levels are U-shaped with a minimum at $60-\mathrm{msec}$ SOA. The -.4 and -.8 accuracy functions are somewhat more complex, particularly the -.4 function. However, both contain a significant quadratic component and reach a minimum at 120 -msec SOA. The -1.6 accuracy function is statistically flat (i.e., the apparent rise is not consistent across subjects).

Except for the .0 function, all of the bias functions are primarily monotonic. The differences in minima for the .0 accuracy and bias functions supports the observation made by Bernstein et al. (1974) that sub- 
Table 1

Trend Analyses for Individual Masking Functions

\begin{tabular}{|c|c|c|c|c|c|c|c|}
\hline \multirow[b]{2}{*}{ Mask } & \multirow[b]{2}{*}{ Measure } & \multicolumn{2}{|c|}{ Main Effect of SOA } & \multicolumn{2}{|c|}{ Linear Component } & \multicolumn{2}{|c|}{ Quadratic Component } \\
\hline & & $\mathbf{F}$ & MSe & $F$ & $\%$ Var & $\mathrm{F}$ & $\%$ Var \\
\hline .0 & Accuracy & $5.02^{*}$ & .0038 & $<1$ & $<1$ & $16.86^{* *}$ & 83 \\
\hline-.2 & Accuracy & $4.37^{*}$ & .0049 & $<1$ & $<1$ & $14.37 * *$ & 82 \\
\hline-.4 & Accuracy & 2.98 & .0047 & $<1$ & $<1$ & $4.93 *$ & 41 \\
\hline $\begin{array}{r}.8 \\
-\quad .8\end{array}$ & Accuracy & $6.40 * *$ & .0014 & $<1$ & $<1$ & $9.29 *$ & 36 \\
\hline-1.6 & Accuracy & 1.10 & .0074 & 1.89 & 56 & 1.25 & 37 \\
\hline $\begin{array}{r}1.0 \\
.0\end{array}$ & Bias & $5.13^{*}$ & .0406 & $6.29^{*}$ & 31 & $11.79 * *$ & 58 \\
\hline-.2 & Bias & 2.38 & .0043 & $5.81^{*}$ & 61 & $<1$ & $<1$ \\
\hline $\begin{array}{r}-.4 \\
-.4\end{array}$ & Bias & $13.22 * *$ & .0031 & $43.14 * *$ & 82 & $9.02 *$ & 17 \\
\hline $\begin{array}{r}-.8 \\
-.8\end{array}$ & Bias & $9.01 * *$ & .0135 & $27.17^{* *}$ & 75 & $5.84^{*}$ & 16 \\
\hline-1.6 & Bias & $7.73^{* *}$ & .0229 & $25.67 * *$ & 83 & $5.07 *$ & 16 \\
\hline
\end{tabular}

Note-df for main effect $=4,12 ; d f$ for trend components $=1,12$.

jects do not necessarily perform most poorly when phenomenal suppression is greatest. This is not an artifact of averaging across contexts, since the minima were at the same SOA for the three .0 functions, the two -.4 functions, and the two -.8 functions prior to averaging.

In short, the analysis of individual masking functions confirms that masking of accuracy occurs for mask energies that are at least $-.8 \log$ units less intense than the test. Just as increasing the intensity of the mask past the point of equal energy with the test causes the masking function to be more monotonic by shifting the minimum to a shorter SOA, decreasing the intensity of the mask seems to cause a shift in the minimum to a longer SOA.

\section{Discriminant Function Analysis}

The LDF analyses performed for each combination of subject, SOA, and luminance range followed - the same procedures used by Bernstein et al. (1976), which, in turn, followed Hake et al. (1967). It should be noted that effects due to the variable labeled "luminance range" are in fact due to physical energy and not to range effects per se. The preliminary analysis conducted to see if the three response centroids were colinear in the decision space indicated that, in fact, they were. The first LDF accounted for $99 \%$ or more of the trace (total variance) in $45 \%$ of the 60 cases and between $95 \%$ and $99 \%$ in an additional $35 \%$ of the 60 cases.

Two measures previously discussed are the coherence, or separation of the centroids along the discriminant, and the accuracy, or separation of the centroids as projected onto the test-energy (relevant) axis. These describe how different the responses are as defined by the subject and as defined by the experimenter's criterion, respectively. Figure 3 contains the mean value of these variables pooled over luminance range, since this was not a significant source of variance. The main effect of SOA was significant for both variables, $F s(4,12)=4.56$ and
7.76 , ps $<.05$ and .01 , for coherence and accuracy, respectively. The linear components accounted for $6 \%$ and $0 \%$ of the variance, $\operatorname{Fs}(1,12)=1.10$ and $<1.0$, both n.s., for these two functions, respectively. The quadratic component accounted for $73 \%$ and $95 \%$ of the variance, $\mathrm{Fs}(1,12)=13.24$ and 29.62 , ps $<.01$. These U-shaped functions replicate what was obtained previously. Contrary to what we usually find, performance at 0 -msec SOA was not superior to the "no-mask" control level.

One of the novel findings in our previous study concerned the slope of the discriminant. Figure 4 contains these slopes, expressed in terms of the angle (in degrees) made between the LDF and the vertical

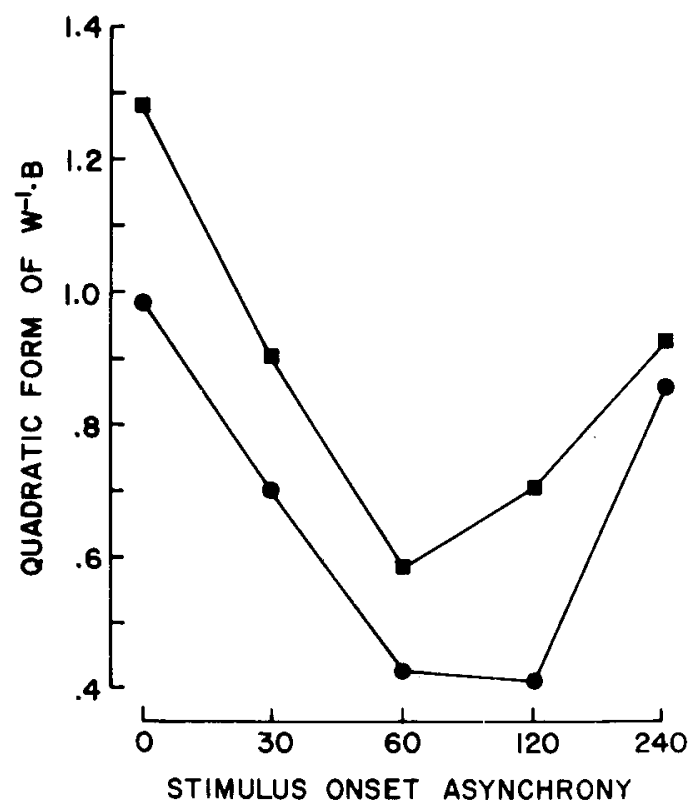

Figure 3. Coherence (squares) and accuracy (circles) pooled over luminance range as a function of stimulus onset asynchrony. (Note-In the "no-mask" control condition only an accuracy measure is obtained, the value of which was 1.17 in this experiment.) 
(relevant) axis, for each luminance range as a function of SOA. Both the narrow- and medium-range conditions produced functions similar to that observed by Bernstein et al. (1976), in that the slopes of the LDFs are negative (subjects reacted to the test/ mask energy difference) at 0 - and $30-\mathrm{msec}$ SOA, became positive (subjects reacted to the test/mask energy sum) at 60 - and $120-\mathrm{msec}$ SOA, and are close to zero (subjects reacted to the test alone) at $240-\mathrm{msec}$ SOA. The wide-range condition produced a similar, but less pronounced, trend. An analysis of variance indicated that the main effect of SOA and the interaction of Luminance Range by SOA were both significant sources of variation, $F(4,12)=6.22$ and $\mathrm{F}(8.24)=4.31$, ps $<.01$.

Finally, we considered goodness-of-fit data in terms of the mean percent variance explained by the two distance models, nondiscriminating and discriminating. The mean difference $(8 \%)$ was very similar to that observed by Bernstein et al. (1976) and was a significant source of variation, $F(1,3)=$ $11.12, \mathrm{p}<.05$. Thus, the discriminating observer model describes the data better than the nondiscriminating observer model. However, the difference was greater at short SOAs than at long SOAs, $F(4,12)$ $=3.50, \mathrm{p}<.05$. These trends are portrayed in Figure 5, pooled over the three luminance range conditions, since this variable did not contribute any significant effects to the analysis.

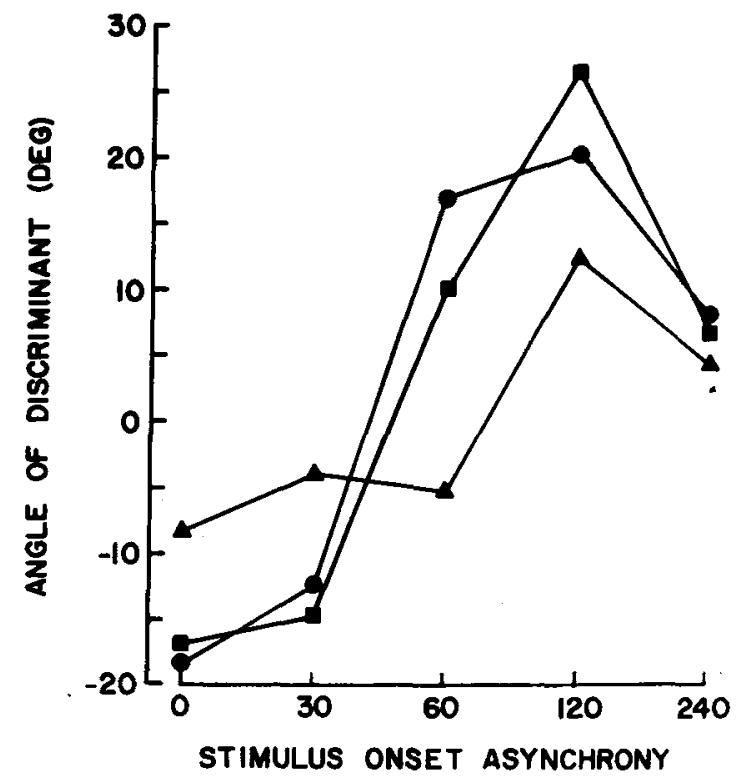

Figure 4. Angle of discriminant (expressed in terms of degrees between discriminant and test-energy axis) pooled over absolute mask energy as a function of luminance range and stimulus onset asynchrony. (Note-Circles $=$ narrow range, squares $=$ medium range, and triangles $=$ wide range.)

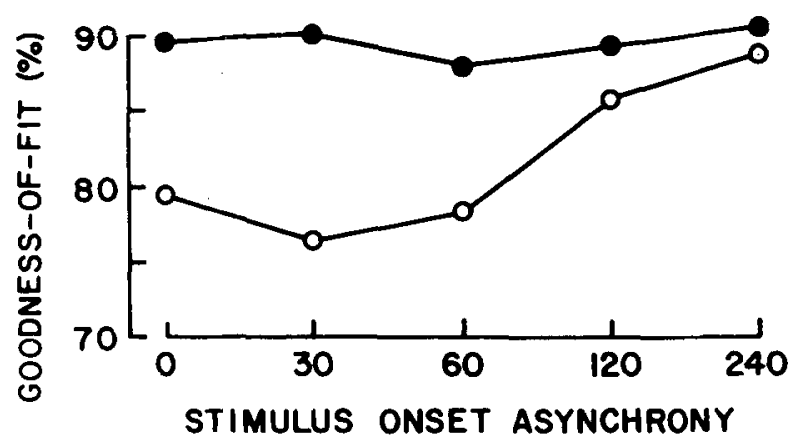

Figure 5. Goodness of fit (expressed in terms of the mean percent variance explained) for the discriminating observer model (filled circles) and the nondiscriminating observer model (open circles) as a function of stimulus onset asynchrony.

\section{DIFFERENCE}

The results in the narrow-range condition are essentially identical to those obtained previously by Bernstein et al. (1976). Furthermore, substantially these same effects were found with the two other energy ranges. Hence, the changes in criterion content with SOA hold when mask energy variation is apparent as well as rather subtle. Consequently, these changes are a pervasive feature of judgments made in a metacontrast situation. And, as discussed by Bernstein et al. (1976), lateral-inhibitory models (Breitmeyer \& Ganz, 1976; Weisstein, 1972) are illequipped to handle these changes in criterion content.

The results obtained with low-intensity masking stimuli generally complement what is usually reported with high-intensity masking stimuli, since we found that decreasing mask intensity decreased the amount of suppression and shifted the minimum point (i.e., maximum suppression) to a longer SOA. The reduction in amount of masking is consistent with Alpern's (1953) low mask-intensity data, but the shift in minimum is not. The finding that the threshold for metacontrast occurs somewhere between -.8 and $-1.6 \log$ units of mask intensity relative to test intensity was also in accord with his findings.

Judgments made on a given trial were almost exclusively the result of the particular stimuli (test and mask) at SOAs of from 0 to $120 \mathrm{msec}$ and the particular test stimulus at the longest SOA $(240 \mathrm{msec})$ rather than the range of stimulus alternatives. In this sense, the results are very different from those presented by Hake et al (1967) in their size judgment task. We have also found fairly substantial context effects of the type discussed by Hake et al. (1967) in a size-distance judgment task using subjects of comparable sophistication to those used in the present experiment. It is not clear why some tasks would lead to these effects and others be immune. 
However, the relative immunity of metacontrast suppression from these effects is, of course, important to demonstrate, given the extensive use of contextually varying masking data in testing quantitative models of metacontrast, such as Weisstein's $(1968,1972)$.

The one measure which did prove context sensitive was the response bias measure (i.e., mean response level). We have previously noted that measures of this type are context-sensitive to SOA. Bernstein et al. (1973) found that subjects tended to adopt the same level of response bias when SOA was held constant over a block of trials but varied this level as a function of SOA when SOA varied from trial to trial. In the former case, subjects matched the stimulus presentation probabilities within each block, whereas in the latter case, they matched the stimulus probabilities for the experiment as a whole.

\section{REFERENCES}

Alpern, M. Metacontrast. Journal of the Optical Society of America, 1953, 43, 648-657.

Bernstein, I. H., Fisicaro, S. A., \& Fox, J. A. Metacontrast suppression and criterion content: A discriminant function analysis. Perception \& Psychophysics, 1976, 20, 198-204.

Bernstein, I. H., Proctor, R. W., Belcher, J., \& Schurman, D. L. An analysis of U-shaped metacontrast. Perception \& Psychophysics, 1974, 16, 329-336.

Bernstein, I. H., Proctor, J. D., Proctor, R. W., \& Shurman, D. L. Metacontrast and brightness discrimination. Perception \& Psychophysics, 1973, 14, 293-297.

Bernstein, I. H., Smith, D. B., \& Adey, M. Perceptual and response interdependencies in visual masking. In S. Dornic \& A. F. Sanders (Eds.), Attention and performance, VI. Potomac, Md: Erlbaum, in press.

BreitmeYer, B., \& GaNz, L. Implications of sustained and transient channels for theories of visual pattern masking, saccadic suppression, and information processing. Psychological Review, $1976,83,1-36$.
GARNER, W. R. The processing of information and structure. Potomac, Md: Erlbaum, 1974.

Hake, H. W., Faust, G. W., McIntyre, J. S., \& Murray, H. G. Relational perception and modes of perceiver operation. Perception \& Psychophysics, 1967, 2, 469-478.

Hake, H. W., \& Rodwan, A. S. Perception and recognition. In J. B. Sidowski (Ed.), Experimental methods and instrumentation in psychology. New York: McGraw-Hill, 1966.

Hake, H. W., Ronwan, A. S., \& Weintraub, D. J. Noise reduction in perception. In K. R. Hammond (Ed.), The psychology of Egon Brunswik. New York: Holt, Rinehart, \& Winston, 1966.

Kahneman, D. Method, findings, and theory in studies of visual masking. Psychological Bulletin, 1968, 70, 404-425.

Lefton, L. A. Metacontrast: A review. Perception \& Psychophysics, 1973, 13, 161-171.

LUCE, R. D. Detection and recognition. In R. D. Luce, R. R. Bush, \& E. Galanter (Eds.), Handbook of mathematical psychology (Vol. 1). New York: Wiley, 1963.

Rodwan, A. S., \& Hake, H. W. The discriminant function as a model for perception. American Journal of Psychology, 1964, 77, 380-392.

WEISSTEIN, N. A. A Rashevsky-Landahl neural net: Simulation of metacontrast. Psychological Review, 1968, 75, 494-521.

WeIssteIN, N. A. Metacontrast. In D. Jameson \& L. M. Hurvich (Eds.), Handbook of sensory physiology (Vol. 7, Part 4, Visual psychophysics), Berlin: Springer-Verlag, 1972.

\section{NOTES}

1. The original intention was to randomly select the sessions during which the control trials would be presented, with the constraint that one control block be presented during each of the three luminance range conditions. Unfortunately, due to miscommunication between the second author and one of the experimeters, only the latter half of the intention was realized.

2. When there are only two alternatives, other response measures such as Luce's (1963) eta and beta measures are preferable on theoretical grounds. When there are three alternatives, however, there are no generally accepted measures derived from a formal theory which are clearly superior to those used.

(Received for publication February 3, 1977; revision accepted June 21,1977 .) 